\title{
16. Relation between Angiographic Tumor Vascularity and Patho-histological Findings of Meningioma
}

\author{
Takayoshi Nomura, Hiroshi Miyagawa and Inazo Toda \\ Neurological Center, National Hospital of Nagoya \\ Toshio Matsubara \\ Dept. of Neurology, Neurosurgery and Psychiatry, \\ Okehazama Hospital
}

Some kinds of meningioma often demonstrate a certain characteristic pictures in cerebral angiograms. The characteristic tumor vascularities so far observed in meningioma are:

(i) visualization of tumor capillaries (so-called tumor stain),

(ii) visualization of smaller tumor vesseles, and

(iii) no visualization of tumor vascular structures.

Authors had some special considerations on the relation between angiographic characteristics and patho-histology of meningioma. In 19 cases of verified meningioma in our series, 5 showed tumor capillaries (tumor stain), 5 visualized smaller tumor vesseles and the remaining 9 had no tumor vascular structures in cerebral angiograms. The marked tumor stain was seen in a case of angiomatous type of meningioma, and moderate tumor stain was encountered in 2 cases of fibromatous (fibrils abundant) type of meningioma, and slight tumor stain was observed in 2 cases of mixed fibromatous (fibrils scanty)-endotheliomatous type of meningioma. All of 5 cases which visualized smaller tumor vesseles were mixed type of fibromatous (fibrils scanty)-endotheliomatous meningioma. Authors have found out that the fibroblastic structures might play significant roles in the $\mathrm{x}$-ray visualization of vascular structures including capillaries and smaller vesseles of inside of meningioma. The remaining 9 cases which visualized no vascular structures were endotheliomatous meningioma.

In conclusions derived from the above short series, angiographic characteristics of inside of meningioma, the visualization of tumor capillaries and smaller tumor vesseles might be related with angioblastic, fibroblastic, and fibroblastic component-mixed type of meningioma, and it is not always related with the closed vascular distribution in the connective tissue of the tumor. No endotheliomatous meningioma showed tumor vascular structures in angiograms. Finally, it was noted that the bleeding during the operative procedures had no relation with the angiographic tumor vascularity. 